\title{
Reversal of Immunogenicity of Hepatitis B Antigen by Treatment with Nutraceuticals (OMEGA QGEL)
}

\author{
Ram B Singh $^{1}$, Miki Tokunaga ${ }^{2}$, Toru Takahashi ${ }^{3}$, Douglas W Wilson ${ }^{4} *$, Elena Gerasimova ${ }^{5}$ and \\ Fabien De Meester ${ }^{5}$
}

\author{
${ }^{1}$ Halberg Hospital and Research Institute, Moradabad, India \\ ${ }^{2}$ Department of Nutrition and Health Sciences, Fukuoka Women's University, Fukuoka, Japan \\ ${ }^{3}$ Graduate School of Human Environment Science, Fukuoka Women's University, Japan \\ ${ }^{4}$ School of Medicine, Pharmacy and Health, Durham, UK \\ ${ }^{5}$ The Tsim Tsoum Institute, Krakow, Poland
}

\begin{abstract}
Two patients presented with positive hepatitis B virus antigen reactivity. Both the patients had medical records indicating clinical and biochemical manifestations of viral hepatitis. Treatment with coenzyme Q10 and w-3 fatty acids was associated with reversal of antigenicity causing negative hepatitis B antigen reactivity.
\end{abstract}

Keywords: Viral hepatitis, antioxidant, liver damage, infection.

\section{INTRODUCTION}

Hepatitis B is an important global health problem with 350 million individuals affected worldwide [1-3]. Approximately, two billion people have been infected with hepatitis $\mathrm{B}$ virus (HBV), 360 million have chronic infection, and 600,000 die each year from HBV-related liver disease or hepatocellular carcinoma, worldwide [4]. The prevalence of HBV infection in India and many other parts of Asia-Pacific regions appears to be quite high [2]. It has been estimated that more than one million children run a lifetime risk of becoming chronic carriers and about 0.1 million die from hepatitis virus complications yearly. An estimated 15 to $40 \%$ patients with hepatitis virus infection would develop complications during the course of hepatitis B virus infection [3]. The hepatitis B virus is present in high concentration in blood, serum, serous exudates, saliva, semen, vaginal fluid and most body fluids [3]. The most important mode of transmission is perinatal transmission in regions with high prevalence rates. However, among adolescents, sexual transmission is the predominant route in developed countries and in low prevalence areas. Approximately $25 \%$ of the babies who are infected with hepatitis B virus, subsequently develop severe chronic liver disease including liver cancer [1-3]. The population of India is approximately 1.2 billion, of which $30 \%$ are carriers of hepatitis B virus. It has been estimated that hepatitis B virus may be responsible for about $68 \%$ cirrhosis and $80 \%$ of cancer in India [3]. The awareness

\footnotetext{
*Address correspondence to this author at the School of Medicine, Pharmacy and Health, Durham University, UK; Tel: + 4419133 40825; Fax: +44 19133 40346; E-mail: dww@tsimtsoum.net
}

about hepatitis B virus infection and its vaccine is believed to be very poor in most developing countries. Therefore, it is important to find out carriers of hepatitis virus and treat them before they infect others for prevention of hepatitis B virus infection. Patients with mild disease and normal alanine transaminase (ALT) may not require immediate treatment and should be carefully monitored at appropriate intervals. Most clinicians feel that therapy should only be considered if there is evidence of moderate to severe activity, as well as disease progression. However, nutraceuticals may be used to prevent and treat immuno-suppression caused by hepatitis B infection. Since, coenzyme Q10 (CoQ10) and w-3 fatty acids are known to enhance immunity, in the present study, we report on the role of CoQ10 in combination with w-3 fatty acids among two patients who had positive hepatitis $\mathrm{B}$ virus antigen.

\section{CASE REPORTS}

CASE 1. A 35 years old male patient presented with weakness, loss of appetite, mild fever, yellow eyes and urine of about 1 week duration. On physical examination, his body weight was $56 \mathrm{~kg}$ and body mass index was $22.3 \mathrm{~kg} / \mathrm{M}^{2}$. Clinical examination revealed; pulse 81 beats/ min, blood pressure $122 / 80 \mathrm{~mm} \mathrm{Hg}$, temperature $37^{\circ} \mathrm{C}$, with yellow conjunctiva. Systemic examination revealed tenderness in the right hypochondrium on palpation, indicating tender liver. Blood examination revealed normal total and differential leucocyte counts, serum bilirubin $5.4 \mathrm{mg} / \mathrm{dl}$, serum glutamic-pyruvic transaminase (SGPT) $345 \mathrm{IU}$ and reactive hepatitis B antigen. 
He was advised rest, B vitamins, appetizers tonic and Lornithine oxoglutarate 2 tablets twice daily for about 4 weeks. There was an improvement in his clinical condition as well as in biochemical parameters in the next 2-3 weeks but his hepatitis $B$ antigen remain reactive at 4 weeks and 6 weeks. His hepatitis B antigen became nonreactive after 16 weeks. Repeated examination of blood for hepatitis B antigen showed negative titers at 20, and 24 weeks and after one year.

CASE 2. A 23 year male patient presented mild fever, weakness, loss of appetite of about 10 days duration with history of jaundice, and higher serum bilirubin, SGPT and reactive hepatitis B antigen, about 4 weeks ago. Physical examination revealed absence of jaundice, oedema, pallour or lymphadenopathy. Systemic examination showed tenderness in the right hypochondrium.

His blood examination revealed positive hepatitis B antigen and normal serum bilirubin and SGPT measured in two laboratories. He was administered L-ornithine oxoglutarate, 2 tablets twice daily. After 24 weeks his blood was nonreactive for hepatitis B antigen along with coenzyme Q10.

Both patients had higher blood levels of IL-6, IL-1 and TNF-alpha and lower CoQ10. Both patients received softgel (product code\# 9634-SEG, Tishcon Corporation, Westbury, NY) containing $600 \mathrm{mg}$ total omega-3 fatty acids (as ethyl esters), EPA $350 \mathrm{mg}$, DHA $180 \mathrm{mg}$, other w-3 fatty acids 70mg and CoQ10 30mg, vitamin E 30mg; each. Each patient was administered two softgel thrice daily for 24 weeks. Total intake of omega-3 fatty acids was $3600 \mathrm{mg} /$ day and CoQ10 $180 \mathrm{mg} /$ day, vitamin E $180 \mathrm{mg} /$ day.

\section{DISCUSSION}

The disappearance of hepatitis B surface antigen (HBs$\mathrm{Ag}$ ) from the serum, the development of anti-HB antibodies, and normalization of liver function may not reflect complete virological recovery from acute hepatitis $B$ virus (HBV) infection [5-7]. It has been demonstrated by polymerase chain reaction (PCR), indicating long-term persistence of HBV DNA in the serum and peripheral blood mononuclear cells (PBMC) of four patients for up to 70 month after complete clinical, biochemical, and serological recovery from acute viral hepatitis. HBV DNA was also present in PBMC in late convalescent samples from all four patients, and HBV RNA was detected in late convalescent phase PBMC in two of these patients. It is possible that $\mathrm{HBV}$ DNA, and possibly HBV virions, can be present in the serum, and that the viral genome can persist in a transcriptionally active form in PBMC for $>5 \mathrm{yr}$ after complete clinical and serological recovery from acute viral hepatitis. We observed that treatment of viral hepatitis B infection followed by nutraceutical administration was associated with nonreactivity of the hepatitis B antigen in serum of our two patients. However, it is quite possible that HBV DNA may be detected among both patients in the next 5 years indicating some activity of the virus $[6,7]$.

Occult hepatitis $\mathrm{B}$ virus (HBV) infection is defined by the presence of HBV DNA in the liver tissue of individuals who test negative for hepatitis B surface antigen ( $\mathrm{HBsAg}$ ) [6]. There is evidence that many patients who have recovered from acute hepatitis B can carry HBV genomes for a long time and show histological patterns of mild necroinflammation, even fibrosis, years after the resolution of acute hepatitis, without showing any clinical or biochemical evidence of liver disease. Occult HBV may be inoffensive itself, in conditions of immunocompetence, but when other relevant causes of liver damage are present it may worsen the course of the liver disease. The risk of HBV transmission through transfusion is related to blood donations negative for HBAg that have been collected during the pre-seroconversion period or during chronic occult $\mathrm{HBV}$. The role of prior hepatitis $B$ immunization with an optimal anti-HBs response in cases of HBV transmission through organ transplantation is not known if it can effectively modulate or abort the infection. Use of antiviral agents as prophylaxis in patients with serological evidence of past HBV infection prevents reactivation of occult hepatitis $B$ virus infection (OBI) after transplantation in most cases. Reactivation of OBI has been observed in other conditions that cause immunosuppression, in which antiviral therapy could be delayed until the HBV DNA or HBsAg becomes detectable. Occult HBV might contribute to the progression of liver fibrosis and hepatocellular carcinoma development in patients with chronic liver disease. Use of HBV nucleic acid amplification testing and multivalent anti-HBs antibodies in the HBsAg assays is recommended for detection of true and false OBI, respectively.

It is widely believed that the hepatitis B virus (HBV) is completely cleared by antiviral antibodies and specific cytotoxic T lymphocytes (CTLs) during acute viral hepatitis [5]. Traces of HBV are often detectable in the blood for many years after clinical recovery from acute hepatitis, despite the presence of serum antibodies and $\mathrm{HBV}-$ specific cytotoxic $\mathrm{T}$ lymphocytes, which can be present at acute-stage levels. The strength of these lymphocytes response to $\mathrm{HBV}$ following clinical recovery correlates with persistence of HBV DNA. $\mathrm{HBV}$-specific lymphocytes from patients studied up to 23 years after clinical and serological recovery expressed activation markers (HLA-DR, CD69) indicating recent contact with antigen. These findings suggest that agents which improve immunity may be administered for a long time to protect against HBV DNA and cytotoxic T lymphocytes response [5-7]. However, in appropriately selected hepatitis B "e" antigen (HBeAg)-positive and $\mathrm{HBeAg-negative} \mathrm{patients,}$ sustained suppression of viral replication can be achieved, and $\mathrm{HBeAg}$ or even HBsAg seroconversion can be attained by interferon alpha $[8,9]$. Nucleoside analogs are given by mouth, once per day, and the safety, potency and efficacy have improved and facilitated treatment. The main advantages of interferon alpha, over nucleoside analogs are the absence of resistance and the possibility of immunemediated clearance of hepatitis B. However, adverse effects preclude the use of interferon alpha in substantial proportions of patients, and prolonged maintenance therapy to suppress hepatitis B virus (HBV) is not feasible. In general, treatment of chronic hepatitis B should target patients with active disease and viral replication, preferably before the signs and symptoms of cirrhosis or significant injury has occurred. Serum aminotransferase levels, serum HBV DNA levels and histological grade and stage are important guiding biomarkers for this treatment. Both forms 
of treatment have benefits and the choice should be selected and tailored. Stopping or futility rules can be implemented in patients who fail interferon. Recent data suggest the safety and efficacy of nucleoside analogs used in the third trimester of pregnancy to reduce the risk of transmission from mothers to their children.

Natural killer cell immunity may be impaired with advanced age, which may have increased susceptibility due to nutritional deficiencies [10-13]. Most patients with nutrient deficiency have an impaired cell-mediated immunity, as well as decreased microbicidal activity of immune cells and increased susceptibility to infections [10-13]. In one study, blood concentrations of selected micronutrients were studied in 62 healthy subjects, aged 90 to 106 years, $(25$ men, 37 women) to find out the association of natural killer cell immunity with nutrient deficiency [13]. The prevalence of micronutrient deficiency was highest for selenium (in $\approx 50 \%$ of both sexes), zinc (in $52 \%$ of men and $41 \%$ of women), and vitamin B-6 (in $40 \%$ of men and $59 \%$ of women), followed by vitamin A (in $16 \%$ of men and $27 \%$ of women) and vitamin $\mathrm{E}$, vitamin B-12, and folate (each in $<10 \%$ of both sexes). Coenzyme Q-10 status was inadequate in $40 \%$ of women and $24 \%$ of men $(P=0.02)$. In women only, natural killer cell cytotoxicity at different effector-target cell ratios was positively associated with plasma vitamin $\mathrm{E}$ and coenzyme Q-10 concentrations $(\mathrm{P}<0.05)$. The study also confirms the high prevalence of micronutrient deficiencies in healthy and apparently well-nourished persons aged $\geq 90 \mathrm{y}$.

Coenzyme Q (CoQ or ubiquinone) is a lipid-soluble component of virtually all types of cell membranes and has been shown to play multiple metabolic functions. Several clinical diseases including encephalomyopathy, cerebellar ataxia and isolated myopathy were shown to be associated with CoQ deficiency. Since CoQ10 is important in the generation of cellular energy, it is not surprising that its deficiencies can cause suppression of the immune system. The role of CoQ10 as an immunomodulating agent has been established in many experimental models which evaluated various parameters of immune function; phagocytic rate, antibody level, cancer, viral and parasitic infections. The liver is quite rich in CoQ10 and hence there may be a loss of CoQ10 due to liver damage during viral hepatitis resulting into CoQ10 deficiency. It is possible that in most infective diseases and in some of the immunity related conditions, CoQ10 may be administered for its bioenergetic immunomodulating activity. The lack of toxicity, as demonstrated in clinical and experimental studies, indicates that CoQ10 is an appropriate candidate for clinical application in disease states where the immune system is not functioning at an optimal level. It is known that CoQ10 is an important component, probably at the mitochondrial level, for the optimal function of the immune system. However, the role of $\mathrm{CoQ}$ in immunity has not been defined. In a recent study, the flies defective in CoQ10 biosynthetic gene coq2 were more susceptible to bacterial and fungal infections, while were more resistant to viruses [10]. In this study, Drosophila contained both CoQ9 and CoQ10, and food supplement of CoQ10 could partially rescue the impaired immune functions of coq2 mutants. Surprisingly, wild-type flies fed CoQ10 became more susceptible to viral infection, which suggested that extra caution should be taken when using CoQ10 as a food supplement. It was also shown that CoQ10 was essential for normal induction of anti-microbial peptides and amplification of viruses. This study determined CoQ content in Drosophila and described its function for the first time in immunity.

Apart from CoQ10, w-3 fatty acids also have immunomodulating activity [10-12]. It is the w-3 fatty acids which possess the most potent immunomodulatory activities, and among the w-3 fatty acids, those from fish oileicosapentaenoic acid (EPA) and docosahexaenoic acid (DHA)-are more biologically potent than alpha-linolenic acid (ALA). Some of the effects of w-3 fatty acids are brought about by modulation of the amount and types of eicosanoids made, and other effects are elicited by eicosanoid-independent mechanisms, including actions upon intracellular signalling pathways, transcription factor activity and gene expression. Recent studies indicate that w-3 fatty acids have anti-inflammatory properties and, therefore, might be useful in the management of inflammatory and autoimmune diseases. Chronic diseases; coronary disease, major depression, aging and cancer are characterized by an increased level of interleukin 1 (IL-1), a proinflammatory cytokine. Similarly, arthritis, Crohn's disease, ulcerative colitis and lupus erythematosis are autoimmune diseases characterized by a high level of IL-1 and the proinflammatory leukotriene LTB produced by omega- 6 fatty acids. Inflammation is part of the normal host response to infection and injury. However, inappropriate inflammation contributes to several diseases, including inflammatory bowel disease (IBD) and rheumatoid arthritis (RA). Both conditions are characterized by the excessive production of inflammatory cytokines, arachidonic acid (AA)-derived eicosanoids, and other inflammatory agents (e.g., reactive oxygen species, adhesion molecules). There have been a number of clinical trials assessing the benefits of dietary supplementation with fish oils in several inflammatory and autoimmune diseases in humans which revealed significant benefit, including decreased disease activity and a lowered use of antiinflammatory drugs $[11,12]$. A large body of evidence also supports a protective effect of omega-3 fatty acids in experimental animal and ex-vivo models of Crohn's disease, ulcerative colitis and rheumatoid arthritis. Most convincing data support the efficacy of $\mathrm{w}-3$ fatty acids in reducing pain, number of tender joints, duration of morning stiffness, use of non-steroidal anti-inflammatory drugs and improving physical performance in rheumatoid arthritis patients. Further clinical trials with large sample size are needed to clarify the efficacy of omega-3 fatty acids as a treatment for other chronic diseases with impaired immunity. In view of the above beneficial effects of CoQ10 and w-3 fatty acids in proinflammatory autoimmune diseases, it is possible that these agents can be protective in all other conditions where natural immunity is impaired. Since hepatitis B antigen reactivity may be associated with impaired immunity and deficiency of immunomodulating nutrients, it is possible that these agents can alter immunity status causing a decrease in antigen reactivity as observed in our patients. In a study involving 63 patients with $\mathrm{HBV}$-associated hepatic carcinoma who underwent hepatectomy, omega-3 fatty acid supplemented total parenteral nutrition $(\mathrm{n}=32)$ for a period of at least 5 days, was found to be associated with lower infection rate [14] 
(omega-3 FA $19.4 \%$ vs. control, 43.8\%). They also had better liver function after hepatectomy: alanine transaminase, aspartate transaminase, total bilirubin, and a shorter length of hospital stay, as compared to a control group who received standard total parenteral nutrition $(\mathrm{n}=31)$. Serum contents of IL-6 and TNF-alpha after hepatectomy were significantly lower in the omega-3 fatty acid group, as compared to the control group. It is possible that administration of omega-3 fatty acid may reduce infection rate and improve liver function recovery in $\mathrm{HBV}$-associated hepatic carcinoma patients after heaptectomy. This improvement is associated with suppressed production of proinflammatory cytokines in these patients. The cellular immune responses, particularly specific cytotoxic

T lymphocytes (CTL), are significant in the host response to HIV - 1 infection [15]. It has been observed that CTL develop quite early in acute HIV - 1 infection, coincident with a rapid fall in plasma virus load, whereas in chronic infection their levels are inversely related to viral load. However the potent HIV-specific CTL response ultimately fails to control HIV replication. Therefore, the immune response and possible causes of depletion of CD4+ T-cell counts in patients with human immunodeficiency (HIV)-1 infection are well known [15].

Hepatitis B virus can be transmitted vertically, through sexual or household contact, or by unsafe injections, but chronic infections acquired during infancy or childhood account for a disproportionately large share of worldwide morbidity and mortality, therefore prevention of infection is more important than treatment. Many developing nations have recently initiated universal hepatitis $\mathrm{B}$ immunization programs with assistance from the Global Alliance for Vaccines and Immunization. Vaccination against hepatitis B virus infection can be started at birth and provides long-term protection against infection in more than $90 \%$ of healthy people [4]. Further progress towards the elimination of hepatitis B transmission will require sustainable vaccination programs with improved vaccination coverage, practical methods of measuring the impact of vaccination programs, and targeted vaccination efforts for communities at high risk of infection. Effects of immunomodulating nutrients on hepatitis B antigen reactivity and on immunity provided by vaccination would need further studies to establish the role of nutritional status in immunity.

\section{ACKNOWLEDGEMENTS}

Acknowledgements are due to Mr Raj Chopra, Tishcon Corporation, Westbury, NY, USA and International College of Nutrition for providing test agents and logistic support to conduct this study.

\section{CONFLICT OF INTEREST}

The authors confirm that this article content has no conflicts of interest.

\section{REFERENCES}

[1] Lok AS, McMahon BJ. Chronic hepatitis B. Hepatology 2007; 34: 507-39.

[2] Liaw YF, Leung N, Guan R, et al. Asian Pacific consensus update working party on chronic hepatitis B. Asian Pacific Consensus Statement on the management of chronic hepatitis B: A 2005 update. Liver Int 2005; 25: 472-89.

[3] Ray SK. Vaccine Preventable diseases. In: Chaturvedi S, Jena TK, Eds. Epidemiology in maternal and child health preventive Medicine. MME-103, New Delhi: IGNOU 2003; pp. 40-66.

[4] Shepard CW, Simard EP, Finelli L, Fiore AE, Bell BP. Hepatitis B Virus, Infection, epidemiology and vaccination. Epidemiol Rev 2006; 28: 112-25.

[5] Rehermann B, Ferrari C, Pasquinelli C. The hepatitis B virus persists for decades after patients' recovery from acute viral hepatitis despite active maintenance of a cytotoxic T-lymphocyte response. Nat Med 1996; 2: 1104-8.

[6] Lledó JL, Fernández C, Gutiérrez ML, Ocaña S. Management of occult hepatitis B virus infection: An update for the clinician. World J Gastroenterol 2011; 17(12): 1563-8.

[7] Michalak TI, Pasquinelli C, Guilhot S, Chisari FV. Hepatitis B virus persistence after recovery from acute viral hepatitis. Clin Invest 1994; 93(1): 230-9.

[8] Dusheiko G. Treatment of HBeAg positive chronic hepatitis B: interferon or nucleoside analogs. Liver Int 2013; 33(Supple1): 137 50 .

[9] Deinstag JL. Hepatitis B infection. N Engl J Med 2008; 359: 1486500 .

[10] Cheg W, Song C, Anjum KM, et al. Coenzyme Q plays opposing roles on bacteria/fungi and viruses in Drosophila innate immunity. Int J Immunogenet 2011; 38: 331-7.

[11] Simopoulos AP. Omega-3 fatty acids in inflammation and autoimmune diseases. J Am Coll Nutr 2002; 21(6): 495-505.

[12] Ruggiero C, Lattanzio F, Lauretani F, Gasperini B, AndresLacueva C, Cherubini A. Omega-3 polyunsaturated fatty acids and immune-mediated diseases: inflammatory bowel disease and rheumatoid arthritis. Curr Pharm Des 2009; 15(36): 4135-48.

[13] Ravaglia G, Forti P, Maioli F, Bastogli L, et al. Effect of micronutrient status on natural killer cell immune function in healthy free-living subjects aged $\geq 90 \mathrm{y}$. Am J Clin Nutr 2000; 71: 590-8.

[14] Wu Z, Qin J, Pu L. Omega-3 fatty acid improves the clinical outcome of hepatectomized patients with hepatitis B virus (HBV)associated hepatocellular carcinoma. J Biomed Res 2012; 26: 3959.

[15] Mishra S, Dwivedi SP, Dwivedi N, Singh RB. Immune Response and Possible Causes of CD4+T-cell Depletion in Human Immunodeficiency Virus (HIV) - 1 Infection. Open Nutraceuticals J 2009; 2: 46-51.

This is an open access article licensed under the terms of the Creative Commons Attribution Non-Commercial License (http://creativecommons.org/licenses/by-nc/3.0/) which permits unrestricted, non-commercial use, distribution and reproduction in any medium, provided the work is properly cited. 